\title{
JOURNAL

\section{Applied horizontal force increases impact loading in reduced-gravity running}

\author{
Young-Hui Chang*, Chris M. Hamerski, Rodger Kram \\ Locomotion Laboratory, Department of Integrative Biology, University of California, Berkeley, CA 94720-3140, USA
}

Accepted 14 September 2000

\begin{abstract}
The chronic exposure of astronauts to microgravity results in structural degradation of their lower limb bones. Currently, no effective exercise countermeasure exists. On Earth, the impact loading that occurs with regular locomotion is associated with the maintenance of bone's structural integrity, but impact loads are rarely experienced in space. Accurately mimicking Earth-like impact loads in a reduced-gravity environment should help to reduce the degradation of bone caused by weightlessness. We previously showed that running with externally applied horizontal forces (AHF) in the anterior direction qualitatively simulates the highimpact loading associated with downhill running on Earth. We hypothesized that running with AHF at simulated reduced gravity would produce impact loads equal to or greater than values experienced during normal running at Earth gravity. With an AHF of $20 \%$ of gravity-specific body weight at all gravity levels, impact force peaks increased $74 \%$, average impact loading rates increased $46 \%$, and maximum impact loading rates increased $89 \%$ compared to running without any AHF. In contrast, AHF did not substantially affect active force peaks. Duty factor and stride frequency decreased modestly with AHF at all gravity levels. We found that running with an AHF in simulated reduced gravity produced impact loads equal to or greater than those experienced at Earth gravity. An appropriate AHF could easily augment existing partial gravity treadmill running exercise countermeasures used during spaceflight and help prevent musculoskeletal degradation. (C) 2001 Elsevier Science Ltd. All rights reserved.
\end{abstract}

Keywords: Biomechanics; Exercise countermeasure; Space flight; Microgravity; Ground reaction force

\section{Introduction}

Chronic exposure to microgravity degrades the structural integrity of the lower extremity bones (for reviews see Baldwin et al., 1996; Vernikos, 1996). This could have catastrophic consequences during mission operations requiring even modest amounts of strength, during emergency escape procedures, and can also increase the risk of fracture after returning to Earth (Baldwin et al., 1996). An effective countermeasure to prevent this degradation of bone is essential for longterm human exploration of space (West, 2000). Although the exact mechanism that stimulates bone formation is not known, it is well established that bone responds to the frequency, rate, distribution, and magnitude of mechanical loading (Burger and Klein-Nulend, 1999; Lanyon, 1996; Rubin and Lanyon,

\footnotetext{
*Corresponding author. Present address: Department of Physiology, Emory University, Atlanta, GA 30322, USA.

E-mail address: younghui@yahoo.com (Y.H. Chang).
}

1984; Whalen, 1993). It seems that some minimum mechanical signal is necessary for the active maintenance of bone architecture (Frost, 1983; Lanyon, 1996). The mechanical loading of bone that occurs with normal activity (i.e., functional loading) is considered to be the primary stimulus (or minimum mechanical signal) for bone to maintain its structural integrity (Frost et al., 1998; Lanyon, 1996).

Lanyon (1996) suggested that any exercise regimen designed to conserve bone mass should utilize high strains imposed at high strain rates. Many others agree that it is essential that an exercise countermeasure provide high-frequency bone strain rates such as those created by the passive impact of the foot with the ground during normal running on Earth (Baldwin et al., 1996; Cavanagh et al., 1992; Greenleaf et al., 1989). Previous exercise countermeasures emphasizing the magnitude of forces actively generated by the muscles have been inadequate in preventing or reducing skeletal degradation (Baldwin et al., 1996; Convertino, 1996; Davis and Cavanagh, 1993; Greenleaf et al., 1989). Simulating 
Earth-like impact loads would provide the natural stress distribution normally experienced by the lower limbs, which would likely preserve the normal distribution of bone as well as its density.

To simulate Earth-like loading conditions during running in space, McCrory and colleagues (1999) tested a motorized treadmill combined with a special harness to apply downward forces to the shoulders and hips of the astronaut. Due to the discomfort of the harness, however, astronauts were unable to use the harness at high enough loads to replicate Earth-like impact loads as evidenced by the ground reaction forces (McCrory et al., 1999). A successful exercise countermeasure involving locomotion must achieve functional mechanical loading of the lower limbs and be comfortable enough for regular daily exercise (Convertino, 1996; McCrory et al., 1999).

We reasoned that a means to increase impact loading during running in simulated partial gravity might allow astronauts to run comfortably while experiencing Earthlike impact loading. Hamill and colleagues (1984) observed that impact peaks increased with downhill grade during running. In vivo tibial bone strain rates during a variety of locomotory tasks were also seen to be greatest during downhill running (Burr et al., 1996). In space there is no downhill, but, running with a forward-pulling applied horizontal force (AHF) is qualitatively similar to downhill running since one must decelerate against a constant force to maintain a constant speed. Chang and Kram (1999) observed that impact force peaks were greater for AHF running at $1 g$. If AHF running produced high-impact loads and strain rates in low gravity, then running with an artificial gravity harness loaded to comfortable levels combined with AHF could be an effective countermeasure. We hypothesized that for treadmill running at simulated reduced gravity, AHF would increase the impact force peaks and impact loading rates to equal or greater values than those experienced during normal running at Earth gravity. Furthermore, a secondary aim of this study was to characterize the vertical ground reaction forces during AHF running in reduced gravity.

\section{Methods}

We collected data on four men and four women between the ages of 19 and $37 \mathrm{yr}$ old $(25.5 \pm 5.6 \mathrm{yr}$, mean $\pm \mathrm{SD}$ ). Subjects gave their informed consent before participating in this study as per the University of California Committee for the Protection of Human Subjects. Body mass of subjects ranged from 48.6 to $80.1 \mathrm{~kg}(63.1 \pm 11.2 \mathrm{~kg})$.

To simulate reduced gravity, we applied a nearly constant upward force to the subject's torso near the center of mass via a modified rock climbing harness (Fig. 1). Four straps attached to the harness a light

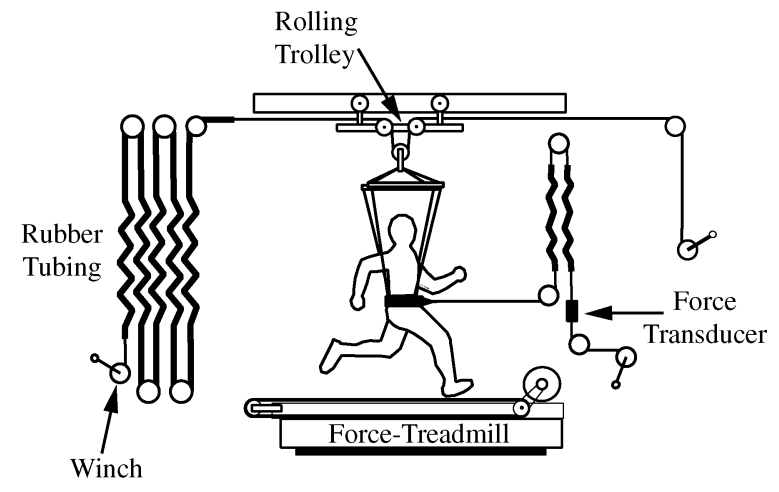

Fig. 1. Schematic of experimental set-up. We simulated reduced gravity by applying a nearly constant upward force to the torso of our subjects near the center of mass. We produced an applied horizontal force (AHF) by pulling the subject forward with a nearly constant force directed near the center of mass. The subjects ran on a force treadmill that measured the vertical ground reaction forces.

aluminum frame above the subject's head. The frame kept the straps away from the torso and did not hinder the ability to lean forward when running. A cable led from the frame over a series of pulleys mounted on an overhead rolling trolley. Movement of the rolling trolley was minimal. Further details of the reduced-gravity simulator have been provided previously (Chang et al., 2000; Donelan and Kram, 2000; Griffin et al., 1999).

We directed the applied horizontal force (AHF) near the subject's center of mass via a belt worn at the waist (Fig. 1). AHF levels were adjusted by stretching the elastic rubber tubing such that minor changes in length (due to the movements of the runner) did not substantially change the AHF. We monitored AHF with a force transducer in series with the rubber tubing. Subjects viewed a digital readout of the AHF as they ran and were instructed to adjust their position on the treadmill so as to keep AHF constant at the prescribed force. The height of the apparatus was adjusted so that the AHF was directed horizontally. Chang and Kram (1999) found that this AHF apparatus did not apply a detectable vertical force. Subjects practiced running in the reduced-gravity simulator at several different combinations of gravity and AHF. The entire familiarization process lasted approximately $30 \mathrm{~min}$ and took place within seven days prior to data collection.

During data collection, subjects ran at $3.0 \mathrm{~m} \mathrm{~s}^{-1}$ for all conditions. In addition to normal $1 g$ running, subjects ran with an AHF of $0,10,15$, and $20 \%$ of the weight reached at each new gravity level. That is, we normalized the AHF to each subject's gravity-specific body weight. Thus, units of AHF are measured in "gravityspecific body weights", which we refer to as "\% AHF".

Subjects ran on a treadmill that measured their vertical ground reaction forces (Kram et al., 1998). We sampled data at $1 \mathrm{kHz}$ per channel and collected data 
A

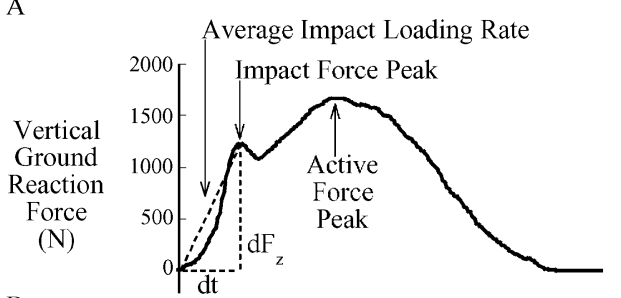

B

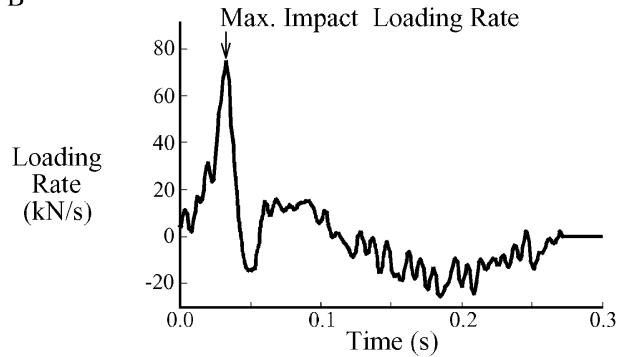

Fig. 2. Typical plots of the vertical component of the ground reaction force (GRF, A) and the loading rate of the vertical GRF (B) versus time for normal $1 \mathrm{~g}$ running. The impact force peak is due to the passive collision of the foot and lower leg with the ground, whereas, the active force peak is associated with the force actively generated by the muscles of the leg. The average impact loading rate was calculated as the slope of the vertical GRF between heel-strike and the impact force peak $\left(\mathrm{d} F_{z} / \mathrm{d} t\right)$. Our notation is that $\mathrm{d} F_{z}$ is the magnitude of impact force peak and $\mathrm{d} t$ is the time to impact force peak.

for 10 steps per trial. We filtered the data with a fourthorder recursive, zero phase-shift, Butterworth low-pass filter $(100 \mathrm{~Hz}$ cut-off). This low-pass cut-off frequency did not attenuate impact force peaks or loading rates. The instant of heel-strike was determined from the vertical force record. Our algorithm found the closest local minimum occurring before the vertical ground reaction force reached a threshold of $100 \mathrm{~N}$. The instant of toe-off was determined by finding the nearest local minimum occurring after the vertical ground reaction force dropped below $100 \mathrm{~N}$.

For each trial, we calculated the impact force peak magnitude, average impact loading rate, maximum impact loading rate, time to impact force peak, active force peak magnitude, duty factor and stride frequency averaged for 10 steps. We determined the impact force peak magnitude by starting from heel-strike and finding the time when the positive slope became negative (Fig. 2A). Average impact loading rate was calculated as the impact force peak magnitude $\left(\mathrm{d} F_{z}\right)$ divided by the change in time $(\mathrm{d} t)$ from heel strike to the impact force peak (Fig. 2A). The maximum impact loading rate was determined by finding the instantaneous derivative of the ground reaction force with respect to time and taking the maximum value during the impact phase of ground contact (Fig. 2B). Time to impact force peak $(\mathrm{d} t)$ was the time from heel strike until impact force peak (Fig. 2A). We determined vertical active force peak in a similar manner but in the reverse direction, starting with toe-off and working backwards in time towards heel strike. Duty factor (time of ground contact for one foot divided by stride time) and stride frequency (inverse of stride time) were calculated from the times of heel strike and toe-off.

We performed a Student's 1-tailed, paired $t$-test to examine whether impact loading values from experimental conditions were significantly lower than control $(1 g$, no AHF). We used a 2-tailed, paired $t$-test for other variables. Statistical significance was defined as $P<0.05$ $(n=8)$. We performed a multivariate regression analysis to estimate the influence of AHF and gravity upon impact force peaks and to predict the appropriate AHF necessary to obtain Earth-like impact loading for a given level of gravity. We used the delta method (for reviews see Goldberger, 1991; Greene, 2000) to determine the standard error curves for each predicted AHF.

\section{Results}

Applied horizontal force (AHF) significantly increased impact force peaks at all levels of gravity (Figs. 3, and 4A, Table 1). At $1 g$ with no AHF (i.e., normal running), impact force peaks were 1.59 times Earth body weight $(\mathrm{EBW})$, or $983 \pm 72 \mathrm{~N}$. At $0.5 \mathrm{~g}$ with no AHF, impact force peaks were only 1.17 times EBW $(724 \pm 74 \mathrm{~N})$. At $0.5 \mathrm{~g}$ with $20 \% \mathrm{AHF}$, however, impact force peaks were 2.04 times EBW $(1260 \pm 107 \mathrm{~N})$. At all levels of gravity, $20 \%$ AHF was sufficient to significantly increase impact force peaks to magnitudes equal to or greater than those observed during normal running at $1 g$ (Fig. 3). Our data for impact force peaks are summarized by the following empirical regression function between impact force peak, gravity and AHF for running at $3 \mathrm{~m} \mathrm{~s}^{-1}$ :

$$
F_{\text {impact }}=0.66+0.91(g)+0.69(\mathrm{AHF})+7.9(g)(\mathrm{AHF}),(1)
$$

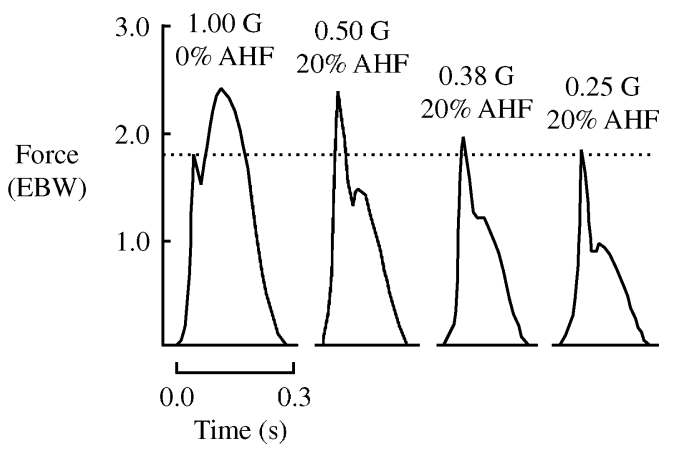

Fig. 3. Typical traces of the vertical component of ground reaction force versus time for the control ( $1 g$, no AHF) condition and for 0.50 , 0.38 , and $0.25 g$ with $20 \%$ AHF. Dotted line indicates the impact force peak for the $1 g$, no AHF condition. Forces are measured in earth body weights (EBW). 
A

B

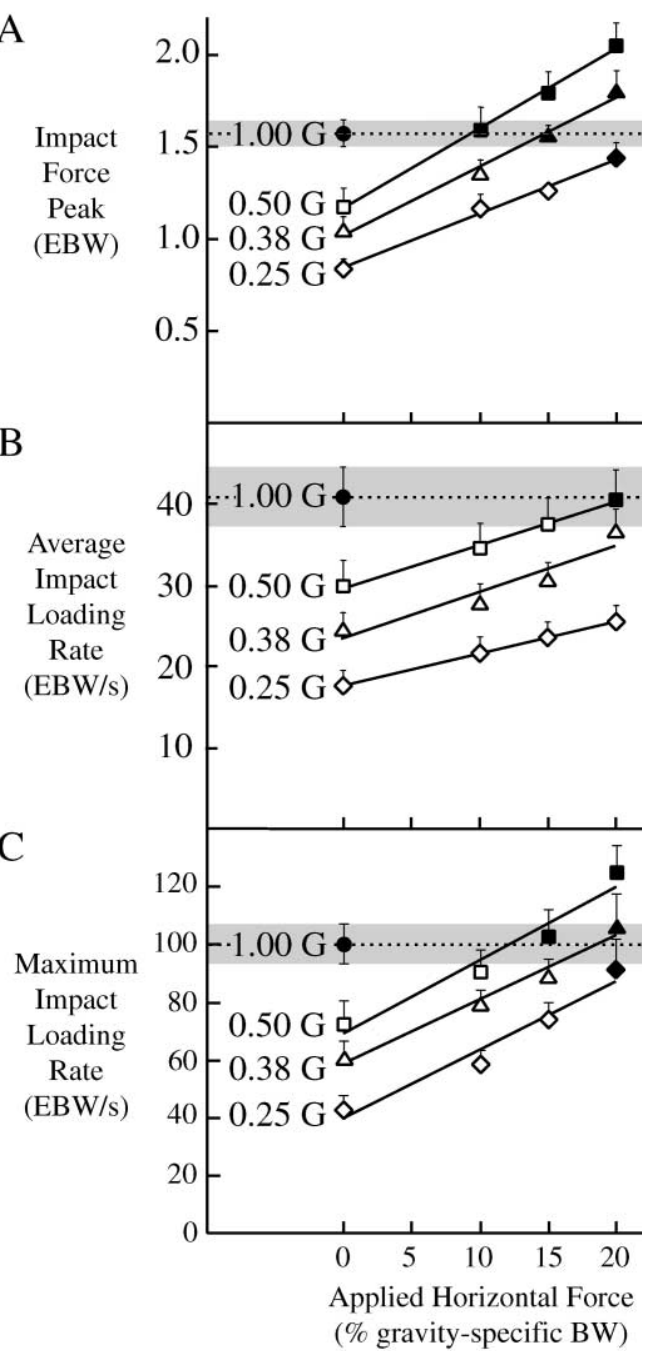

Fig. 4. Impact force peak (A), average impact loading rate (B), and maximum loading rate $(\mathrm{C})$ as functions of applied horizontal force (AHF). Data for Earth gravity, $1 \mathrm{~g}$ (circle and dotted line), $0.50 \mathrm{~g}$ (squares), $0.38 g=$ Mars gravity (triangles), and $0.25 g$ (diamonds) are shown. Data indicate mean for eight subjects and are measured in units of either Earth body weights (EBW) or Earth body weights $/ \mathrm{s}^{-1}$ (EBW $\mathrm{s}^{-1}$ ). Filled symbols indicate that the values were not significantly lower than values for normal running at Earth gravity (1-tailed, paired $t$-test, $p>0.05)$. Shading indicates \pm 1 standard error from the mean of control $(1 g$, no AHF) values. Error bars indicate standard error of the mean.

where $F_{\text {impact }}$ is the impact force peak magnitude in Earth body weights, $g$ is the fraction of Earth gravity, and AHF is the fraction of the gravity-specific bodyweight of applied horizontal force.

An increase in AHF also significantly increased vertical impact loading rates at all levels of gravity (Fig. 4B, C, Table 1). At $1 g$ with no AHF (i.e., normal running), average and maximum impact loading rates were 26.1 and $61.7 \mathrm{kN} \mathrm{s}^{-1}$, respectively. At $0.5 \mathrm{~g}$ with no $\mathrm{AHF}$, average and maximum impact loading rates were reduced to only 18.2 and $44.1 \mathrm{kN} \mathrm{s}^{-1}$, respectively. A
$20 \%$ AHF at $0.5 g$, however, increased the average and maximum impact loading rates to 24.8 and $76.9 \mathrm{kN} \mathrm{s}^{-1}$, respectively. Even at Mar's gravity of $0.38 \mathrm{~g}$, a $20 \%$ AHF increased the average and maximum impact loading rates to normal Earth-like values. These increased loading rates occurred despite an increase in the time to impact force peak. Although reduced gravity alone had no effect on the time to impact force peak, there was a small but significant increase in the time to impact force peak with increasing AHF (Table 2).

Active force peaks during normal running decreased in reduced gravity and were not affected substantially by AHF (Fig. 3, Table 2). During normal running, active force peaks were 2.42 times their Earth body weight (EBW) $(1495 \pm 100 \mathrm{~N})$. At $0.5 \mathrm{~g}$ with no AHF, active force peaks were only $1.37 \mathrm{EBW}(848 \pm 58 \mathrm{~N})$. The maximum increase in active force peaks from all AHF conditions averaged a mere $9 \%$ for each gravity level. This is overshadowed by the $74 \%$ increase in impact force peaks with AHF at each gravity level.

Duty factor and stride frequency both decreased modestly in reduced gravity. The observed changes at different gravity levels were similar to previous reports (Chang et al., 2000; Donelan and Kram, 2000; He et al., 1991). Both duty factor and stride frequency decreased with increasing AHF (Table 2). At all gravity levels, a $20 \%$ AHF decreased both duty factor and stride frequency from zero AHF values by 13.2 and $8.4 \%$, respectively.

\section{Discussion}

As hypothesized, an applied horizontal force (AHF) dramatically increased impact loading during running at all gravity levels. Running with an AHF is, thus, a potentially useful augmentation to existing exercise countermeasures to the bone loss associated with chronic exposure to microgravity. We suggest that with an AHF, a gravity-inducing harness need only load the shoulders and hips of exercising astronauts to partial gravity levels and, thus, could be comfortable enough for regular exercise in microgravity. At Earth gravity with no AHF, the predicted impact force peak at $3 \mathrm{~m} \mathrm{~s}^{-1}$ is 1.6 EBW. Thus, from Eq. (1) we can, solve for the magnitude of AHF needed to produce Earth-like impact loads as a function of $g$ :

$$
\mathrm{AHF}=[0.91(1 g)] /[0.69+7.9(g)] .
$$

This relationship (Eq. (2)) allows us to predict for any level of gravity the minimum AHF necessary to obtain impact force peaks equal to those experienced during normal running on Earth (Fig. 5). For example, at $0.5 \mathrm{~g}$ the equation would predict a minimum of $9.8 \%$ AHF to obtain Earth-like impact loads. 
Table 1

Effects of gravity and AHF on impact loading. Data for running at normal Earth gravity $(1 g)$ and at different simulated reduced gravity levels with applied horizontal forces (AHF). Conditions that effectively mimic the impact loading during normal Earth running are denoted as NSL (not significantly lower, $P>0.05$, 1-tailed, paired $t$-test) than the control (1g, no AHF). Values represent mean and standard error

\begin{tabular}{|c|c|c|c|c|}
\hline Gravity $(g)$ & $\begin{array}{l}\text { Applied horizontal } \\
\text { force }(\% g \text {-specific BW) }\end{array}$ & $\begin{array}{l}\text { Impact force } \\
\text { peak }(\mathrm{N})\end{array}$ & $\begin{array}{l}\text { Average impact } \\
\text { loading rate }\left(\mathrm{kN} \mathrm{s}^{-1}\right)\end{array}$ & $\begin{array}{l}\text { Max. impact loading } \\
\text { rate }\left(\mathrm{kN} \mathrm{s}^{-1}\right)\end{array}$ \\
\hline 1.00 & 0 & $983(72)$ & $26.1(2.4)$ & $61.7(5.4)$ \\
\hline \multirow[t]{4}{*}{0.50} & 0 & 724 (74) & $18.2(1.7)$ & $44.1(4.6)$ \\
\hline & 10 & $979(93)^{\mathrm{NSL}}$ & $21.1(1.9)$ & $55.5(5.7)$ \\
\hline & 15 & $1111(102)^{\mathrm{SL}}$ & $23.1(2.3)$ & $64.1(8.1)^{\mathrm{NSL}}$ \\
\hline & 20 & $1261(107)^{\mathrm{SL}}$ & $24.8(2.4)^{\mathrm{NSL}}$ & $76.9(7.9)^{\mathrm{NSL}}$ \\
\hline \multirow[t]{4}{*}{0.38} & 0 & $640(56)$ & $14.8(1.2)$ & $37.0(3.7)$ \\
\hline & 10 & $835(61)$ & $16.9(1.2)$ & $48.8(4.4)$ \\
\hline & 15 & $965(75)^{\mathrm{NSL}}$ & $18.8(1.5)$ & $55.5(4.9)$ \\
\hline & 20 & $1109(91)^{\mathrm{NSL}}$ & 22.5 (1.9) & $64.5(6.0)^{\mathrm{NSL}}$ \\
\hline \multirow[t]{4}{*}{0.25} & 0 & $510(35)$ & $10.5(1.0)$ & $25.6(2.1)$ \\
\hline & 10 & $712(55)$ & $13.1(1.1)$ & $35.6(3.1)$ \\
\hline & 15 & $772(45)$ & $14.4(1.0)$ & $45.0(3.2)$ \\
\hline & 20 & $889(82)^{\text {NSL }}$ & $15.8(1.6)$ & $55.7(7.0)^{\mathrm{NSL}}$ \\
\hline
\end{tabular}

Table 2

Effects of gravity and AHF on time to impact force peak, active force peak and stride kinematics. Data for running at normal Earth gravity $(1 g)$ and at different simulated reduced gravity levels with applied horizontal forces (AHF). In all but one condition, the time-to-peaks were longer $(P<0.05$, 2 -tailed, paired $t$-test) than the control ( $1 \mathrm{~g}$, no AHF). NS denotes condition that was not significantly $(P>0.05)$ different from the control. All levels of reduced gravity had significantly lower active force peaks, shorter duty factors and lower stride frequencies than the control condition $(P<0.05)$. Values represent mean and standard error

\begin{tabular}{llllll}
\hline Gravity & $\begin{array}{l}\text { Applied horizontal } \\
\text { force }(\% g \text {-specific BW) }\end{array}$ & $\begin{array}{l}\text { Time to impact } \\
\text { force peak }(\mathrm{ms})\end{array}$ & $\begin{array}{l}\text { Active force peak } \\
(\mathrm{N})\end{array}$ & Duty factor & Stride frequency (Hz) \\
\hline 1.00 & 0 & $38(1)$ & $1495(100)$ & $0.35(0.01)$ & $1.37(0.02)$ \\
0.50 & 0 & $42(2)$ & $848(58)$ & $0.30(0.02)$ & $1.19(0.03)$ \\
& 10 & $47(1)$ & $901(77)$ & $0.28(0.01)$ & $1.16(0.03)$ \\
& 15 & $48(2)$ & $890(71)$ & $0.27(0.01)$ & $1.15(0.02)$ \\
0.38 & 20 & $51(1)$ & $865(76)$ & $0.27(0.01)$ & $1.12(0.02)$ \\
& 0 & $43(2)$ & $664(40)$ & $0.28(0.01)$ & $1.17(0.04)$ \\
& 10 & $48(2)$ & $666(49)$ & $0.26(0.01)$ & $1.13(0.03)$ \\
0.25 & 15 & $50(2)$ & $700(60)$ & $0.25(0.01)$ & $1.10(0.03)$ \\
& 20 & $50(2)$ & $715(57)$ & $0.24(0.01)$ & $1.06(0.02)$ \\
& 0 & $47(3)$ & $463(39)$ & $0.26(0.02)$ & $1.11(0.04)$ \\
& 10 & $50(2)$ & $493(33)$ & $0.23(0.01)$ & $1.06(0.04)$ \\
& 15 & $51(3)$ & $513(38)$ & $0.23(0.01)$ & $1.04(0.03)$ \\
\end{tabular}

In addition to spaceflight, our results have implications for extended missions to the surfaces of Mars or the moon. At as low as $0.38 \mathrm{~g}$ with only a $15 \%$ AHF, impact force peaks were equal to or greater than those normally experienced during normal $1 g$ running. We observed that at $0.38 \mathrm{~g}$ with an AHF of $20 \%$ the impact force peaks and maximum loading rate during treadmill running were similar to those normally experienced while running on Earth. Thus, during a long-term mission on the surface of Mars, adequate impact loading of the lower limbs could likely be achieved through AHF running with no other external loads. We found that balance on a treadmill is compromised an AHF that is greater than $20 \%$. Thus, a minimum level of gravity (e.g., $\sim 0.3 g$ ) seems necessary to safely and effectively simulate Earth-like impact loading. Therefore, a hypothetical exercise countermeasure on the lunar surface $(0.17 \mathrm{~g})$ would require a harness to induce some level of increased artificial gravity.

Since the mechanism for stimulating bone formation remains unresolved, it is difficult to assess the relative importance of impact loading vs. the active force peak magnitudes for conserving the structural integrity of bone. AHF running, however, does provide a means for distinguishing between them since AHF substantially affects only impact force peaks and not active force peaks. Further study focusing on the long-term effects of impact vs. active forces as biomechanical determinants of bone remodeling would help determine their 


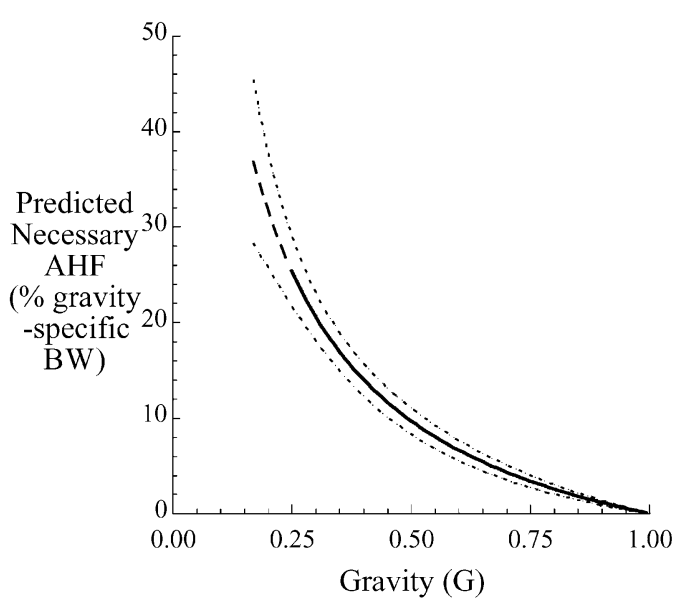

Fig. 5. Predicted AHF necessary to reach Earth-like impact force peaks as a function of gravity level while running at $3 \mathrm{~m} \mathrm{~s}^{-1}$ (calculated from Eq. (2)). Dashed line represents an extrapolation to Lunar gravity $(0.17 \mathrm{~g})$. Dotted lines indicate \pm 1 standard error of the mean as calculated by the delta method (for reviews see Goldberger, 1991; Greene, 2000).

independent roles in influencing bone architecture and strength.

Reduced functional mechanical loading of the skeletal system may not be the proximate mechanism for decreased bone formation at the cellular level. In microgravity, fluid shifts from the lower extremities toward the upper body have also been suggested to be the cause of bone mineral loss. Alterations in skeletal perfusion have been correlated with changes in bone mass in rats undergoing hindlimb unloading experiments (Colleran et al., 2000). That study, however, was unable to distinguish between the effect of mechanical unloading and the shift in fluid perfusion in the limb bones. The two mechanisms may be interrelated. The strain-derived flow of interstitial fluid through the porosity of bone caused by mechanical loading has been observed to activate osteocytes (Burger and KleinNulend, 1999). The loss of bone mass due to skeletal unloading either in microgravity or simulated microgravity is often accompanied by fluid shifts, which has caused some confusion as to the exact stimulus for bone formation.

As Lanyon (1996) points out, functional mechanical loading of the skeletal system is necessary to not only maintain absolute bone mass, but also to preserve the structural integrity of bone. Although the proximate mechanism for bone formation may not be known, AHF running in space may allow humans to maintain bone mass despite the accompanying fluid shifts in microgravity. If this were the case, it would suggest that impact loading should play a critical role in any exercise countermeasure against bone loss.

The increase in impact loads when running with an AHF may be caused by changes in muscle activation and leg geometry (or orientation). The horizontal braking force, which decelerates the mass of the runner within each step, increases with a forward-pulling AHF. The propulsive force, normally generated to accelerate the runner with each step, approaches zero with a forward-pulling AHF (Chang and Kram, 1999). The increased braking forces required to run with a forwardpulling AHF are likely achieved with greater eccentric muscle activation in the leg muscles (as in downhill running), decreased angular excursion at the knee, and higher torsional joint stiffness. These parameters have been observed to increase simulated running impact force peaks in mathematical models (Nigg and Liu, 1999; Gerritsen et al., 1995). Landing velocity is another parameter that greatly influences impact forces during running (Gerritsen et al., 1995). Although the AHF likely increases the horizontal impact force peak due to an increase in horizontal landing velocity, horizontal landing velocity does not have a direct effect on the vertical impact force peak since it does not affect downward forces. As with the vertical impact force peak, the horizontal impact force peaks decreased with reduced gravity, but increased with AHF (unpublished data). Nevertheless, horizontal force peaks did not contribute substantially to the magnitude of the resultant impact forces generated. For example, at $0.5 \mathrm{~g}$ and all AHF levels, the contribution of the horizontal impact force to the resultant impact force was typically less than $6 \%$ (unpublished data).

In the present study, we have shown that impact loads during running can be increased at different gravity levels with a forward-pulling applied horizontal force. This could prove to be a useful augmentation to current exercise countermeasures to prevent the degradation of the structural integrity of bone during chronic exposure to microgravity. We suggest that increased impact loading with AHF is due to the adjustments in muscle activation, joint angles, and torsional joint stiffnesses made by the runner. Although an AHF increases the impact loading experienced in running, it does not affect the active forces generated. This may prove useful for distinguishing the independent effects of impact forces and active forces on mechanisms for conserving the structural integrity of bone.

\section{Acknowledgements}

We thank the members of the University of California at Berkeley Locomotion Laboratory and Julia Fox for their insightful comments and suggestions to the manuscript. We are also grateful to Michael Brunetti and the UC Berkeley Statistical Consulting Services for their assistance with our statistical analyses. This work was supported in part by the University of California, Berkeley Undergraduate Research Apprenticeship 
Program (to C.M.H.), the University of California, Berkeley Biology Fellows Program (to C.M.H.) and NIH R29-AR44688 (to R.K.).

\section{References}

Baldwin, K.M., White, T.P., Arnaud, S.B., Edgerton, V.R., Kraemer, W.J., Kram, R., Raab-Cullen, D., Snow, C.M., 1996. Musculoskeletal adaptations to weightlessness and development of effective countermeasures. Medicine and Science in Sports and Exercise 28, $1247-1253$.

Burger, E.H., Klein-Nulend, J., 1999. Mechanotransduction in bonerole of the lacunocanalicular network. FASEB Journal 13, S101S112.

Burr, D.B., Milgrom, C., Fyhrie, D., Forwood, M., Nyska, M., Finestone, A., Hoshaw, S., Saiag, E., Simkin, A., 1996. In vivo measurement of human tibial strains during vigorous activity. Bone 18, 405-410.

Cavanagh, P.R., Davis, B.L., Miller, T.A., 1992. A biomechanical perspective on exercise countermeasures for long term spaceflight. Aviation Space and Environmental Medicine 63, 482-485.

Chang, Y.-H., Huang, H.-W.C., Hamerski, C.M., Kram, R., 2000. The independent effects of gravity and inertia on running mechanics. Journal of Experimental Biology 203, 229-238.

Chang, Y.-H., Kram, R., 1999. Metabolic cost of generating horizontal forces in running. Journal of Applied Physiology 86, 1657-1662.

Colleran, P.N., Wilkerson, M.K., Bloomfield, S.A., Suva, L.J., Turner, R.T., Delp, M.D., 2000. Alterations in skeletal perfusion with simulated microgravity: a possible mechanism for bone remodeling. Journal of Applied Physiology 89, 1046-1054.

Convertino, V.A., 1996. Exercise as a countermeasure for physiological adaptation to prolonged spaceflight. Medicine and Science in Sports and Exercise 28, 999-1014.

Davis, B.L., Cavanagh, P.R., 1993. Simulating reduced gravity: a review of biomechanical issues pertaining to human locomotion. Aviation, Space and Environmental Medicine 64, 557-566.

Donelan, J.M., Kram, R., 2000. Exploring dynamic similarity in human running using simulated reduced gravity. Journal of Experimental Biology 203, 2405-2415.

Frost, H.M., 1983. A determinant of bone architecture. Clinical Orthopaedics and Related Research 175, 286-292.

Frost, H.M., Ferretti, J.L., Jee, W.S.S., 1998. Perspectives: some roles of mechanical usage, muscle strength, and the mechanostat in skeletal physiology, disease, and research. Calcified Tissue International $62,1-7$.

Gerritsen, K.G., van den Bogert, A.J., Nigg, B.M., 1995. Direct dynamics simulation of the impact phase in heel-toe running. Journal of Biomechanics 28, 661-668.

Goldberger, A.S., 1991. A Course in Econometrics. Harvard University Press, Cambridge, MA, pp. 102, 109-110.

Greene, W.H., 2000. Econometric Analysis. Prentice-Hall, Upper Saddle River, New Jersey, pp. 205-207, 357-358, 718-719.

Greenleaf, J.E., Bulbulian, R., Bernauer, E.M., Haskell, W.L., Moore, T., 1989. Exercise-training protocols for astronauts in microgravity. Journal of Applied Physiology 67, 2191-2204.

Griffin, T.M., Tolani, N.A., Kram, R., 1999. Walking in simulated reduced gravity: mechanical energy fluctuations and exchange. Journal of Applied Physiology 86, 383-390.

Hamill, C.L., Clarke, T.E., Frederick, E.C., Goodyear, L.J., Howley, E.T., 1984. Effects of grade running on kinematics and impact force. Medicine and Science in Sports and Exercise 16, 185.

He, J.P., Kram, R., McMahon, T.A., 1991. Mechanics of running under simulated low gravity. Journal of Applied Physiology 71, 863-870.

Kram, R., Griffin, T.M., Donelan, J.M., Chang, Y.H., 1998. A forcetreadmill for measuring vertical and horizontal ground reaction forces. Journal of Applied Physiology 85, 764-769.

Lanyon, L.E., 1996. Using functional loading to influence bone mass and architecture: objectives, mechanisms, and relationship with estrogen of the mechanically adaptive process in bone. Bone 18 , S37-S43.

McCrory, J.L., Lemmon, D.R., Sommer, H.J., Prout, B., Smith, D., Korth, D.W., Lucero, J., Greenisen, M., Moore, J., Kozlovskaya, I., Pestov, I., Stepansov, V., Miyakinchenko, Y., Cavanagh, P.R., 1999. Evaluation of a treadmill with vibration isolation and stabilization (TVIS) for use on the international space station. Journal of Applied Biomechanics 15, 292-302.

Nigg, B.M., Liu, W., 1999. The effect of muscle stiffness and damping on simulated impact force peaks during running. Journal of Biomechanics 32, 849-856.

Rubin, C.T., Lanyon, L.E., 1984. Regulation of bone formation by applied dynamic loads. Journal of Bone and Joint Surgery. American Volume 66, 397-402.

Vernikos, J., 1996. Human physiology in space. BioEssays 18, 1029 1037.

West, J.B., 2000. Historical perspectives: physiology in microgravity. Journal of Applied Physiology 89, 379-384.

Whalen, R., 1993. Musculoskeletal adaptation to mechanical forces on earth and in space. The Physiologist 36, S127-S130. 